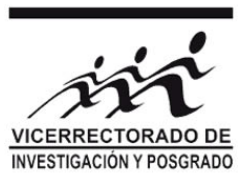

\title{
Estudio de la región de energía prohibida en sólidos con estructura de diamante: Aplicación a Silicio Si
}

\author{
César Cabrera Arista $^{*}{ }^{1,2}$ \\ ${ }^{1}$ Universidad Nacional del Callao, Instituto de Investigación de Ingeniería Química, Callao, Perú \\ ${ }^{2}$ Universidad Nacional Mayor de San Marcos, Facultad de Ciencias Físicas, Ap. Postal 14-0149, Lima, Perú
}

Recibido 15 agosto 2018 - Aceptado 23 noviembre 2018

\begin{abstract}
La energía prohibida del silicio con estructura cristalina de diamante se determinó indirectamente a partir de la estructura electrónica: las bandas de energía y la densidad de estados DOS en el estado fundamental $\mathrm{T}=0^{\circ} \mathrm{K}$. Usando el método de los orbitales lineales Muffin-Tin (LMTO) junto con un potencial efectivo se resolvió la ecuación de Schrödinger del solido y se obtuvo las bandas de energía y la densidad de estados. La energía total mínima de -16.85 Ry por celda unitaria, ocurre para una máxima transferencia de la carga a las esferas vacías en la diagonal de la red cristalina. El gap de energía prohibida asociada es de 0.099 Ry que equivale a $1.35 \mathrm{eV}$, un valor cercano al gap experimental de $1.17 \mathrm{eV}$ que ya existe en la literatura.

Palabras clave: orbitales LMTO, bandas de energía y DOS, red cristalina de diamante.
\end{abstract}

\section{Study of the forbidden energy region in solids with diamond structure: Application to Silicon Si}

Forbidden power of silicon with crystal structure of diamond is determined indirectly from the electronic structure: energy bands and the density of States both in the ground state $T=0^{\circ} \mathrm{K}$. Using the method of the orbital linear Muffin-Tin (LMTO) together with an effective potential of the solid Schrödinger equation was solved and obtained energy bands and the density of States. The minimum total energy -16.85 Ry per unit cell, occurs for maximum transfer of load to empty sphere on the diagonal of the Crystal network. Associated forbidden energy gap is 0.099 Ry which is equivalent to $1.35 \mathrm{eV}$, a value close to the experimental gap of $1.17 \mathrm{eV}$ that already exists in the literature.

Keywords: LMTO orbitals, energy bands and DOS, crystal lattice of diamond.

\section{Introducción}

Conocer las propiedades electrónicas de los semiconductores como el silicio es de gran importancia por su gran potencial de aplicación en nuevas tecnologías y en aplicaciones a escala nanométrica. En décadas recientes en gran parte de los centros de investigación los estudios sobre las propiedades electrónicas de los materiales semiconductores como silicio se hacen con técnicas de simulación en computadoras, como estudio tight-binding y metodo montecarlo de las propiedades electronicas de Si y Ge de D. A. Rasero [1]; la estructura de bandas de semiconductores de N. A. Mecholsky [2]; con técnicas de mucho menor riesgo en comparación con las técnicas experimentales que requieren de equipos sofisticados, de alto costo y con materiales o insumos difíciles de conseguir en su estado altamente puro. En la práctica resulta ventajoso y más económico realizar un primer estudio teórico que

\footnotetext{
${ }^{*}$ ccarista@hotmail.com
}

permita predecir las propiedades electrónicas. El propósito de la presente investigación es mostrar que se pueden calcular las propiedades electrónicas: las bandas de energía, la densidad de estados DOS y la brecha de energía prohibida (gap) de materiales sólidos con estructura cristalina de diamante como el silicio y usar el modelo de C. Cabrera [3. para estudiar los cambios en la brecha (gap) de energía prohibida debido a la transferencia de una parte de la carga electrónica a las regiones vacías de la red cristalina. Aunque existen métodos atomísticos recientes para determinar la estructura electrónica de los materiales semiconductores, resulta conveniente usar la teoria del funcional de la densidad DFT de W. Kohn [4] que usa la aproximacion de la densidad de spin local LDA [5] para el término de intercambio y correlación, que permite formular un potencial efectivo de interacción electrónica, que se requiere para resolver la ecuación auto-consistente de 
Schrödinger para el solido cristalino.

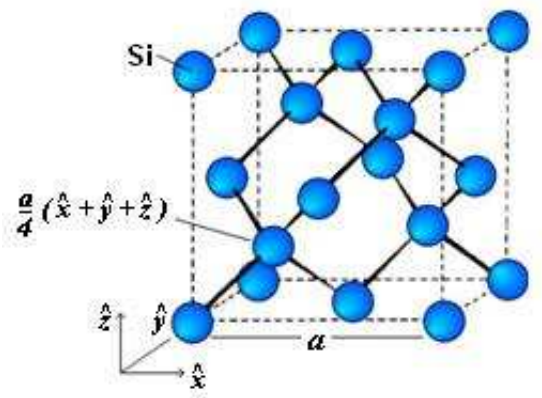

Figura 1: Red cristalina tipo diamante del silicio

\section{Fundamento teórico}

De acuerdo al formalismo teórico del funcional de la densidad de W. Kohn [6] la teoría de la estructura electrónica de la materia se fundamenta en la ecuación no relativista de Schrödinger para un sistema de muchos electrones con función de onda $\Psi$ y energía $E$, que escrita en unidades atómicas Rydberg es:

$\left\{-\frac{1}{2} \sum_{j} \nabla_{j}^{2}-\sum_{j l} \frac{2 Z_{l}}{\left|r_{j}-R_{l}\right|}+\sum_{j \neq i} \frac{1}{\left|r_{j}-r_{i}\right|}\right\}|\Psi\rangle=E|\Psi\rangle$

Sistema con una densidad de carga electrónica $n(r)$, dada por:

$$
n(r)=\sum_{j=1}^{N}\left|\psi_{j}\right|^{2}
$$

El principio variacional de Hohenberg-Kohn [4] establece que si $n(r)$ es la correcta densidad de carga electrónica del estado fundamental, esta minimiza la energía $E$ del sistema. La aplicación del teorema del valor extremo a la ecuación $E=\langle\Psi|H| \Psi\rangle$ con respecto a los orbitales $\psi_{j}(r)$ que están sujetos a la condición de normalidad, se obtiene la ecuación del valor extremo de Euler-Lagrange:

$$
\frac{\partial}{\partial \psi_{j}^{*}(r)}\left\{E-\sum_{j} f_{j} \epsilon_{j} \int\left|\psi_{j}(r)\right|^{2} d^{3} r\right\}=0
$$

En esta ecuación $f_{j} \epsilon_{j}$ son los multiplicadores de Lagrange. Después de efectuar el riguroso cálculo de valores extremos a la ecuación anterior, se obtiene la ecuación de auto-consistente de Kohn-Sham [6] para un electrón que se mueve en un campo de potencial potencial efectivo, escrita en unidades atómicas Rydberg es:

$$
\left\{-\frac{1}{2} \nabla^{2}+V_{e f f}(r)\right\} \psi_{j}(r)=\epsilon_{j} \psi_{j}(r)
$$

El significado de $\epsilon_{j}$ como auto-valor de energía es bien explicado por el argumento de Slater [7] y Janak [8]. El campo de potencial efectivo en el que se mueve el electrón contiene los términos siguientes:

$$
V_{e f f}(r)=v(r)+\int \frac{n\left(r^{\prime}\right)}{\left|r-r^{\prime}\right|} d r^{\prime}+V_{x c}(r)
$$

En la que $V_{x c}(r)$ es el potencial local de intercambio y correlación, con una dependencia funcional de la densidad de carga electrónica $n(r)$. Estas ecuaciones ahora son Ilamadas las ecuaciones auto-consistente de Kohn-Sham [6]. Con estas ecuaciones resueltas la energía del estado fundamental se puede escribir de la forma siguiente:

$$
\begin{aligned}
E= & \sum_{j} \epsilon_{j}+E_{x c}[n(r)]-\int V_{x c}(r) n(r) d r^{3} \\
& -\frac{1}{2} \int \frac{n(r) n\left(r^{\prime}\right)}{\left|r-r^{\prime}\right|} d r^{3}
\end{aligned}
$$

Energía que se puede calcular, puesto que ya se conocen los auto-valores de energía y las funciones de onda al resolver la ecuación auto-consistente de Kohn-Sham [4]. Donde el término $E_{x c}(n(r))$ es la energía potencial de intercambio y correlación, que en la aproximación LDA [5] tiene una dependencia funcional de la densidad de carga electrónica de la forma:

$$
E_{x c}[n(r)]=-0.9305 \int[n(r)]^{\frac{4}{3}} d^{3} r
$$

\section{Datos}

El silicio es un elemento del grupo IVA de la tabla periódica que tiene configuración electrónica de valencia $3 s^{2} 3 p^{2}$. En su estado fundamental $\left(\mathrm{T}=0^{\circ} \mathrm{K}\right.$ ) este material adopta una fase solida con estructura cristalina cubica de tipo diamante, con una constante de red [9] de $5.43 \AA$, red cristalina abierta con regiones en la diagonal del cristal sin ocupación atómica como se aprecia en la figura (1).

Para el cálculo del potencial efectivo $V_{\text {eff }}(r)$ se usan esferas atómicas con un radio $R_{w s}=2.527$ a.u. (unidad atómica), potencial que usaremos para resolver la ecuación de Kohn-Sham.

\section{Metodología}

El cálculo de las bandas de energía y la densidad de estados DOS del silicio se efecctúa usando el método de los orbitales LMTO-ASA [10] para resolver la ecuación auto-consistente de Kohn-Sham [4 6] para el electrón que interacciona con un potencial efectivo que ya contiene toda la información del solido cristalino y calculado en la 
aproximación LDA [3 5]. La energía total se determina resolviendo la ecuación (6) pues la densidad de carga electrónica $n(r)$ y la DOS ya son conocidas después de resolver la ecuación de Kohn-Sham.

El potencial efectivo de interacción del electrón con la red cristalina del solido, que usamos para resolver la ecuación auto-consistente de Kohn-Sham, es un potencial de tipo muffin-tin que depende de la densidad de carga electrónica que a su ves es transferidad a la red cristalina mediante un parámetro $\gamma$ que varía entre $0.0 \leq \gamma \geq 1.0$. Usando el potencial de correlación de un gas homogéneo de electrones, reportado por McLaren [11] en 1991, como aproximación para el término de intercambio y correlación en el potencial efectivo se resuelve la ecuación de Kohn-Sham del solido cristalino y se obtienen los parametros potenciales. Conocidos los parámetros potenciales se construye la base ortogonal de orbitales LMTO [12], que usamos para resolver la ecuación de Schrödinger y calcular la estructura de las bandas de energía y la densidad de estados (DOS) del sistema.

\section{Bandas de Energía, $\mathbf{g}=\mathbf{0 . 0}$}

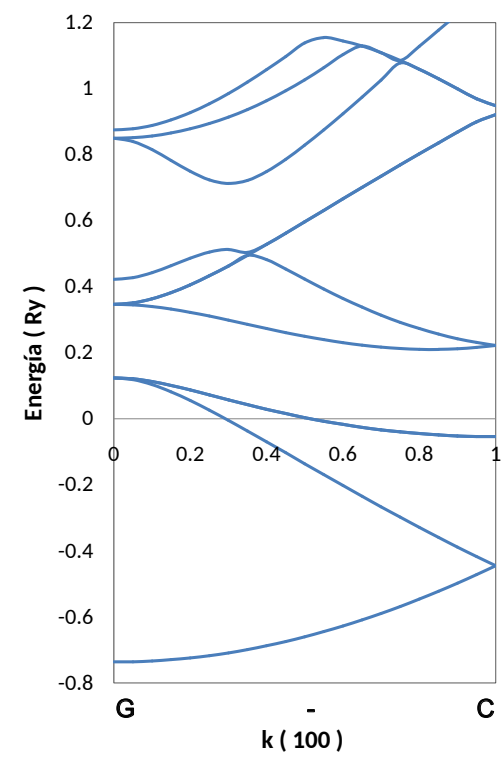

Figura 2: Bandas de energía del Silicio, calculadas para $\gamma=0.0$

\section{Resultados y discusión}

La ecuación de Schrödinger para el sólido cristalino de silicio se resuelve en espacio $k$ junto con la base ortogonal de los orbitales LMTO y se obtiene la relación de dispersión $\epsilon=\epsilon(k)$, que técnicamente constituye la estructura de las bandas de energía del sistema. El cálculo de las bandas de energía se efectúa en la dirección cristalográfica (100) y para algunos valores del parámetro $\gamma$. Las bandas de energía calculadas para $\gamma=0.0$ esto es ninguna transferencia de la carga electrónica a las regiones vacías de la red, se presenta en la Figura 1, la gráfica muestra un buen perfil con una brecha de energía prohibida indirecta de $E_{\text {gap }}=0.115 \mathrm{Ry}$, que equivale a $1.564 \mathrm{eV}$ un poco mayor a lo reportado.

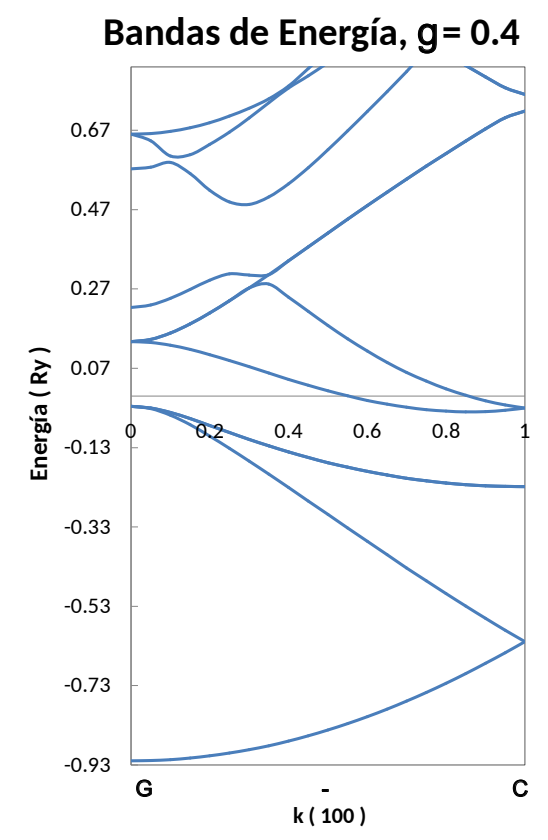

Figura 3: Bandas de energía del Silicio, calculadas para $\gamma=0.4$

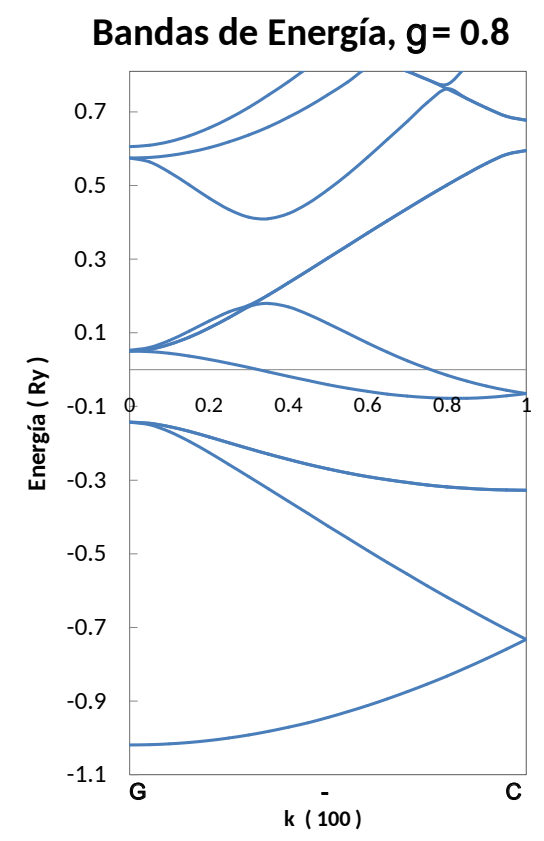

Figura 4: Bandas de energía del Silicio, calculadas para $\gamma=0.8$ 
La brecha de energía se reduce a $E_{\text {gap }}=0.026$ Ry $=$ $0.36 \mathrm{eV}$ para $\gamma=0.2$ una transferencia del $20 \%$ de la carga electrónica a la red cristalina. En la Figura 3 la gráfica muestra el perfil de las bandas de energía con una pequeña brecha de energía prohibida de $E_{\text {gap }}=0.027$ Ry $=0.367$ eV calculada para $\gamma=0.4$ una transferencia del $40 \%$ de la carga a la red cristalina. Para una mayor transferencia de carga electrónica a la red cristalina como $\gamma=0.6$ las bandas de energía mantienen su perfil pero con una brecha de energía prohibida de mayor valor $E_{g a p}=0.054$ Ry $=0.734 \mathrm{eV}$. Este comportamiento de la energía prohibida se mantiene para los cálculos de las bandas de energía con una mayor transferencia de carga electrónica a la red cristalina. Para $\gamma=0.8$ las bandas de energía muestran una brecha prohibida $E_{\text {gap }}=0.069 \mathrm{Ry}=0.938 \mathrm{eV}$ como se aprecia en la Figura 4.

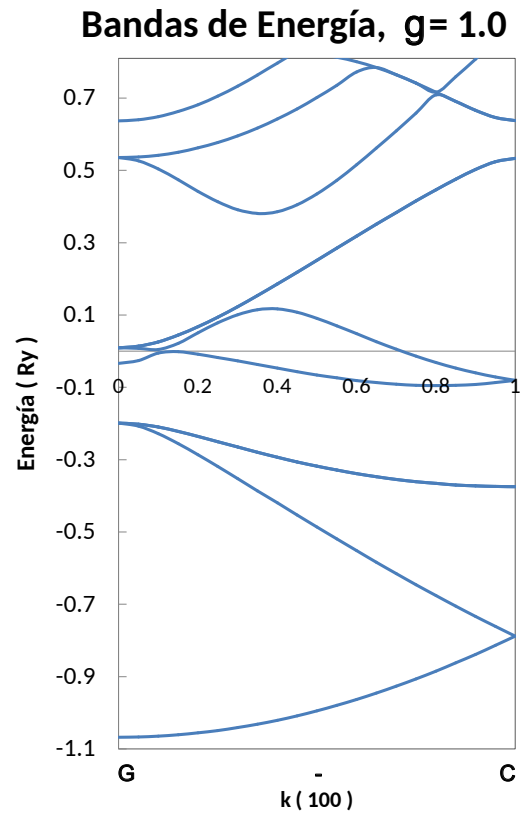

Figura 5: Bandas de energía del Silicio, calculadas para $\gamma=1.0$

La estructura de las bandas de energía calculadas con $\gamma=1.0$, esto es para una transferencia del $100 \%$ de la carga electrónica a la red cristalina, presenta un excelente perfil con una brecha de energía prohibida $E_{\text {gap }}=0.099$ $\mathrm{Ry}=1.35 \mathrm{eV}$, las bandas se han desplazado hacia menores valores de energía como se aprecia la Figura 5.

Los cálculos de la densidad de estados (DOS) presentan un buen perfil y están de acuerdo a los resultados obtenidos para las bandas de energía. La Figura 6 muestra la DOS en función de la energía calculada con $\gamma=0.0$, la gráfica muestra una brecha de energía prohibida en regiones positivas de energía. La densidad de estados calculada para $\gamma=0.2$ presenta una menor brecha de energía prohi- bida, confirmando los resultados de las bandas de energía calculadas para el mismo valor de $\gamma$. La Figura 7 muestra la DOS calculada con $\gamma=0.4$ la gráfica muestra un buen perfil y confirma la pequeña región de energía prohibida obtenida en los cálculos de las bandas de energía.

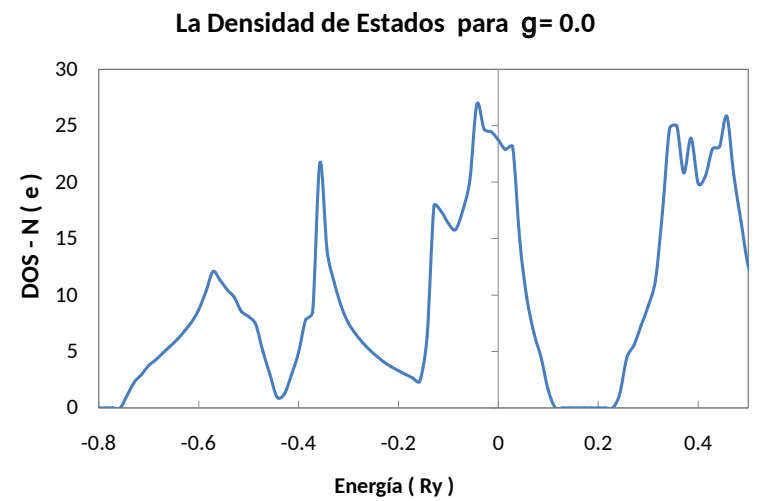

Figura 6: Densidad de estados del Silicio, calculadas para $\gamma=0.0$

\section{La Densidad de Estados para $\mathrm{g}=0.4$}

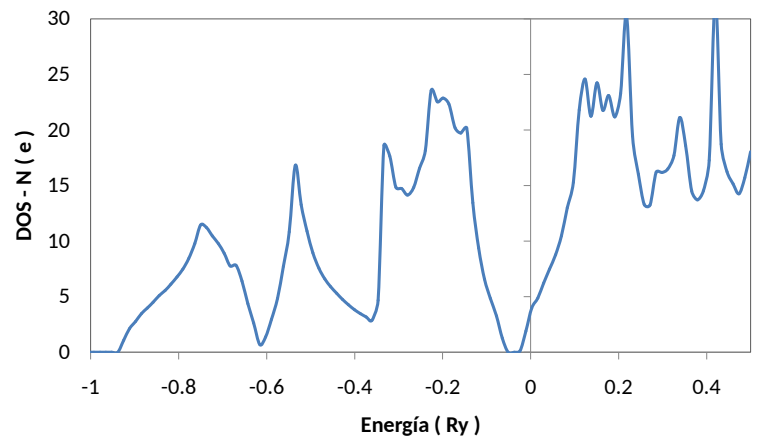

Figura 7: Densidad de estados del Silicio, calculadas para $\gamma=0.4$

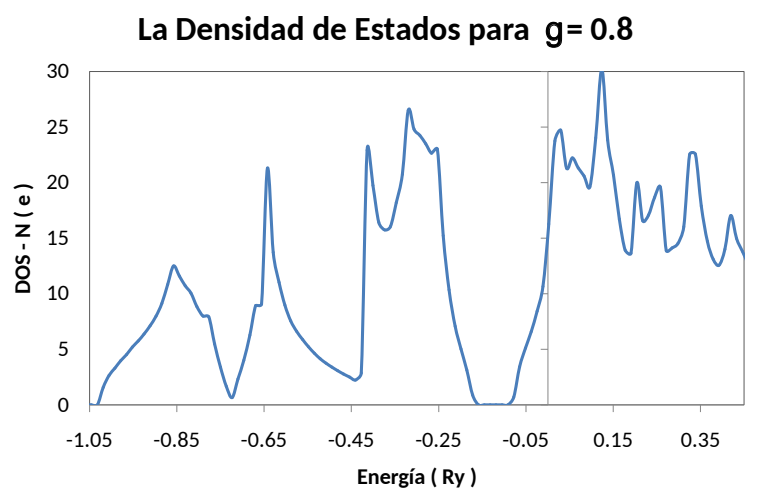

Figura 8: Densidad de estados del Silicio, calculadas para $\gamma=0.8$ 


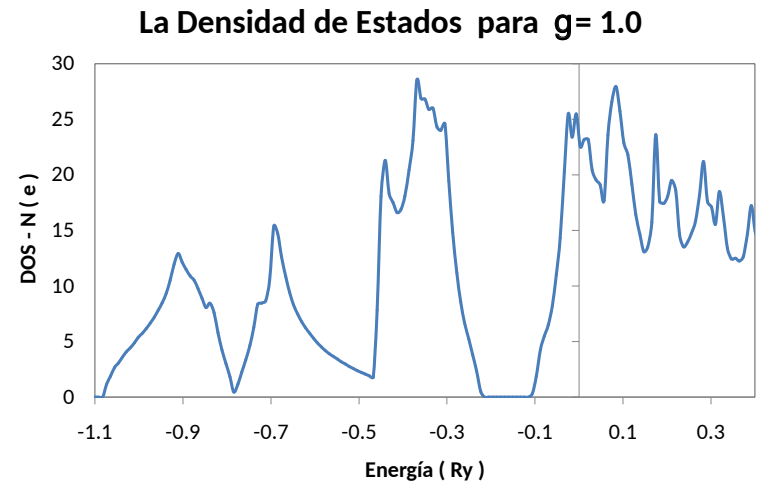

Figura 9: Densidad de estados del Silicio, calculadas para $\gamma=1.0$
La Figura 8. muestra la densidad de estados calculada con $\gamma=0.8$, esto es para una transferencia del $80 \%$ de la carga electrónica a las esferas vacías de la red cristalina, como se puede ver se mantiene el perfil de la DOS calculadas para los otros valores de $\gamma$ pero se observa una mayor brecha de energía prohibida, que confirma lo ya obtenido en las bandas de energía calculadas para el mismo valor de $\gamma$. La densidad de estados calculada con $\gamma=1.0$ una transferencia del $100 \%$ de la carga electrónica a las esferas vacías de la red cristalina, presenta un excelente perfil, con una región de energía prohibida indirecta como se obtuvo en las bandas de energía.

La región de energía prohibida obtenida a partir de la densidad de estados calculadas para los diferentes valores de $\gamma$ se muestran en la tabla 1 que se muestra a continuación.

\begin{tabular}{lcccccr}
\hline$\gamma$ & 0.0 & 0.2 & 0.4 & 0.6 & 0.8 & 1.0 \\
\hline$E_{\text {gap }}(R y)$ & 0.115 & 0.026 & 0.027 & 0.054 & 0.069 & 0.099 \\
$E_{\text {gap }}($ a.u $)$ & 1.56 & 0.36 & 0.37 & 0.73 & 0.94 & 1.35 \\
\hline
\end{tabular}

Tabla 1: Energía prohibida del silicio calculadas para diferentes valores de $\gamma$

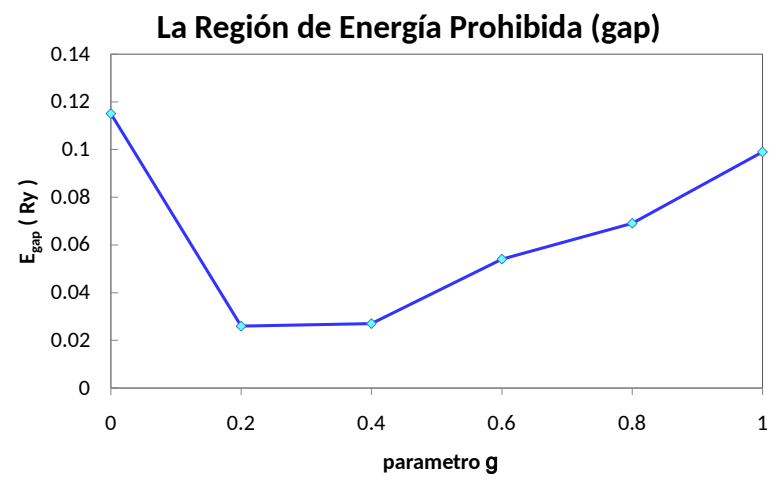

Figura 10: Brecha de energía prohibida en función de $\gamma$

La energía total del estado fundamental calcula para los diferentes valores de $\gamma$ el parámetro de transferencia de la carga electrónica a las esferas vacías de la red cristalina se muestra en la tabla 2. La energía total es de -16.29 Ry para $\Gamma=0.0$ una nula transferencia de carga a las esferas vacías de la red, con una brecha de energía prohibida de $E_{\text {gap }}=0.115 \mathrm{Ry}=1.56 \mathrm{eV}$ mostrando su carácter semiconductor. Los resultados con $\Gamma=1.0$, que corresponde a una transferencia del $100 \%$ de la carga electrónica a las esferas vacías de la red, muestran que la energía total del sistema es mínima con un valor de $-16.85 \mathrm{Ry}$, con una brecha de energía prohibida de $0.099 \mathrm{Ry}=1.35 \mathrm{eV}$, cercano al valor experimental de $1.17 \mathrm{eV}$ reportado en la literatura [13]. La Figura 5 muestra la energía total en función de $\gamma$.

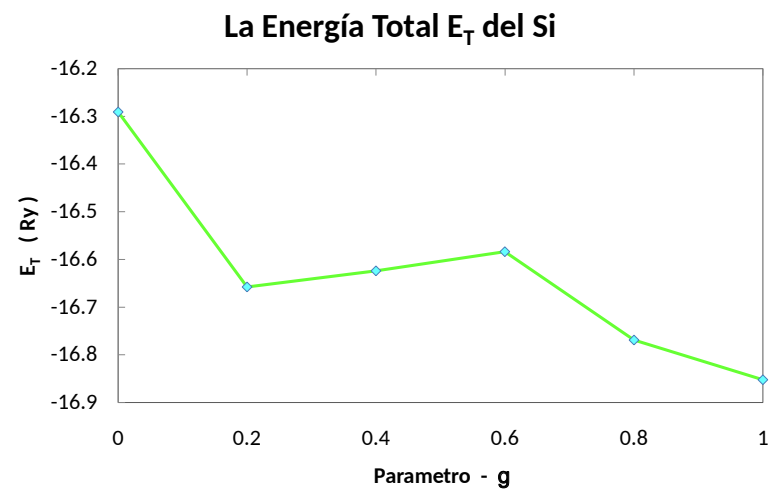

Figura 11: Energía Total del silicio en función de $\gamma$

\section{Conclusiones}

Las bandas de energia y la densidad de estados DOS calculadas con $\gamma=1.0$ una máxima transferencia de la carga electrónica a las esferas vacías de la red cristalina, presentan un excelente perfil, que se ajusta muy bien a los resultados obtenidos por C. S. Wang [13] ó de J. A. Majewsky [14] usando los orbitales LCGG y LDA respectivamente. En la DOS calculada para $\gamma=1.0$ la banda de energía prohibida queda bien definida con un valor de 0.099 Ry, aproximadamente $1.35 \mathrm{eV}$, que es del orden al valor experimental de $1.17 \mathrm{eV}$ reportado en [13], y com- 
parable al valor reportado en otras investigaciones con diferentes métodos [1]. La energía total de -16.85 Ry para $\gamma=1.0$, la máxima transferencia de la carga electrónica a las esferas vacías de la red cristalina, es la que minimiza la energía total del silicio, que implica una mayor estabilidad del sistema.

\section{Agradecimientos}

Mis sinceros agradecimientos al Instituto de Investigación de la Facultad de Ingeniería Química - Universidad Nacional del Callao, por su continuo apoyo a la investigación en la materia condensada.

\begin{tabular}{lcccccr}
\hline$\gamma$ & 0.0 & 0.2 & 0.4 & 0.6 & 0.8 & 1.0 \\
\hline$E_{\text {gap }}(R y)$ & -16.29 & -16.66 & -16.62 & -16.58 & -16.77 & -16.85 \\
$E_{\text {gap }}(\mathrm{eV})$ & -221.56 & -226.55 & -226.09 & -225.54 & -228.06 & -229.19 \\
$E_{\text {Fermi }}(R y)$ & -0.114 & -0.016 & -0.051 & -0.100 & -0.159 & -0.214 \\
$E_{\text {Fermi }}(\mathrm{eV})$ & -1.55 & -0.217 & -0.694 & -1.360 & -2.160 & -2.91 \\
\hline
\end{tabular}

Tabla 2: La energía total, la energía de Fermi del silicio calculadas para diferentes valores del coeficiente $\gamma$

\section{Referencias}

[1] D. A. Rasero; Estudio mediante tight-binding y método Montercalo de las propiedades electrónicas de Si y Ge, Rev. Col. de Fisica. Vol. 413 (2009).

[2] N. A. Mechoslky; Electronic structure for fourteen semiconductors of the diamond and zinc-blende structures, Electronic address: nmech@vsl.cua.edu; URL: http://ape.umd.edu(2011).

[3] C. Cabrera Arista, and H. J. Nowak; Uso de la corrección de auto-energía en el cálculo de la estructura electrónica de sólidos cristalinos Facultad de Ciencias Físicas, UNMSM, Lima Peru (1996).

[4] P. Hohenberg and W. Kohn; Physics Review B. 136, 864 (1964).

[5] J. P. Perdew, and A. Zunger; Spin Density Functional Theory Physical Review B. 30, 5048 (1980).

[6] W. Kohn; Nobel lecture: Electronic Structure of matter-wave function and density functionals Rev. Mod. Phys. 5, vol. 71, 1253 (1999).

[7] J. C. Slater; The self consistent field for molecules and solids McGraw Hill, New York, 1 (1974).
[8] J. F. Janak; Physcal Review B, 140, 1133 (1965).

[9] N. Aschroft; Solid State Physics, Holt Rinehart and Wiston, USA, 129 (1976).

[10] H. L. Skriver; The LMTO Method: Muffi-Tin Orbitals and Electronic Structurre Springer-Verlag, Berlin (1984).

[11] J. M. McLaren; Parameterised local spin density exchange-correlation energies and potentials for electronic structure calculations Comp. Phys. Comms. 66, 383-391 (1991).

[12] H. J. Nowak; Conferencias sobre cálculo de la estructura electrónica con el método LMTO Bogota, Colombia (1991).

[13] Y. Wang; Electronic structure of III-V zinc-blende semiconductors from first principles, Physical Review B. 87, 235203 (2013).

[14] J. A. Majewsky; Advances in the theory of electronic structure of semiconductors Phys. Stat. Sol. vol. 81 , 2003-2027 (2004). 